\title{
Bias-Selective Dual-Operation-Mode Ultraviolet Schottky-Barrier Photodetectors Fabricated on High-Resistivity Homoepitaxial GaN
}

\author{
Feng Xie, Hai Lu, Dunjun Chen, Member, IEEE, Fangfang Ren, Rong Zhang, and Youdou Zheng
}

\begin{abstract}
We demonstrate a dual-operation-mode ultraviolet (UV) Schottky-barrier photodetector (PD) fabricated on highresistivity GaN homoepitaxial layer with low defect density. The undoped GaN active layer is grown by metal-organic chemical vapor deposition on a conductive bulk GaN substrate. Under reverse and zero bias, the $P D$ works in depletion mode with low dark current and high UV/visible rejection ratio. Under forward bias, the PD works alternatively in photoconductive mode, which exhibits high photo-responsivity and an attractive narrow detection band around $365 \mathrm{~nm}$. In addition, the PD also shows reasonable response speed in both operation modes.
\end{abstract}

Index Terms-Dual operation mode, GaN, homoepitaxy, Schottky-barrier photodetector.

\section{INTRODUCTION}

$\mathbf{G}$ ALLIUM nitride (GaN)-based semiconductors are very attractive for ultraviolet (UV) photodetector (PD) applications due to their wide tunable direct bandgap, high electron drift velocity, superior radiation hardness and high temperature resistance [1]. Over the last decade, a variety of GaN-based UV detectors, such as photoconductive [2], metal-semiconductor-metal [3], Schottky barrier [4], and $p-i-n$ PDs [5] have been successfully demonstrated with respective advantages. Among these PDs, photoconductive PDs are attractive for their high responsivity and ease of fabrication but are usually associated with slow response and large dark current. Meanwhile, $p-i-n$ or Schottky-type PDs own the advantages of low dark current and potentially high speed but theoretically have no gain. To meet the requirement of UV detection in various application fields, it is highly

Manuscript received June 2, 2012; revised September 12, 2012; accepted September 23, 2012. Date of publication October 2, 2012; date of current version November 28, 2012. This work was supported in part by the State Key Program for Basic Research of China under Grant 2010CB327504, Grant 2011CB922100, and Grant 2011CB301900, in part by the National Natural Science Foundation of China under Grant 60825401, Grant 60936004, Grant 11104130, and Grant 60990311, in part by the Natural Science Foundation of Jiangsu Province under Grant BK2011050 and Grant BK2011556, and in part by the Priority Academic Program Development of Jiangsu Higher Education Institutions.

F. Xie is with the Jiangsu Provincial Key Laboratory of Advanced Photonic and Electronic Materials, and School of Electronic Science and Engineering, Nanjing University, Nanjing 210093, China, and also with the 38th Research Institute of China Electronics Technology Group Corporation, Hefei 230088, China.

H. Lu, D. Chen, F. Ren, R. Zhang, and Y. Zheng are with the Jiangsu Provincial Key Laboratory of Advanced Photonic and Electronic Materials, and School of Electronic Science and Engineering, Nanjing University, Nanjing 210093, China (e-mail: hailu@nju.edu.cn).

Color versions of one or more of the figures in this letter are available online at http://ieeexplore.iee.org.

Digital Object Identifier 10.1109/LPT.2012.2222022 desirable to combine the benefits of different types of PDs into one single device.

In this letter, by fabricating a vertical Schottky-type PD on high resistivity $\mathrm{GaN}$ homoepitaxial layer with low defect density, we first demonstrate a bias-selective dual-operationmode UV PD. Depending on bias polarity, the PD could work in either depletion mode or photoconductive mode with respective attractive performance. The PD also exhibits reasonable response speed in both operation modes.

\section{EXPERIMENT}

The PD is fabricated on freestanding conductive bulk GaN substrate prepared by hydride vapor phase epitaxy with a thickness of $300 \mu \mathrm{m}$ and a room-temperature resistivity of $\sim 0.01 \Omega \cdot \mathrm{cm}$. The device structure consisting of a $1 \mu \mathrm{m}$ silicon doped $n^{+}$GaN $\left(\sim 3 \times 10^{18} \mathrm{~cm}^{-3}\right)$ transition layer and a $3 \mu \mathrm{m}$ undoped $\mathrm{GaN}$ active layer is grown by metal-organic chemical vapor deposition. Based on our past study, the undoped GaN homo-epilayer could be highly resistive under optimized growth conditions [6]. Its thickness is carefully chosen so that the forward dark current of the PD to be reported later could lie below an acceptable level. The dislocation density of the GaN homo-epilayer is measured by a cathodoluminescence (CL) mapping technique, which has been confirmed to be a reliable method to count dislocations in low-dislocation-density GaN [7]. Fig. 1(a) shows a typical panchromatic CL mapping image of the GaN homo-epilayer examined over a large surface area, in which each dislocation is represented by a small dark spot resulting from strong local non-radiative recombinations. The dislocation density of the GaN homoepilayer is estimated to be $5 \times 10^{6} \mathrm{~cm}^{-2}$ in average, which is about $2-3$ orders lower than the typical dislocation density of hetero-epitaxial GaN. The corresponding full width at half maximum (FWHM) of the GaN (0002) XRD rocking curve is $\sim 80-100$ arcsec, which agrees with the low dislocation density determined by CL mapping.

To fabricate the vertical Schottky-type PDs, a back Ohmic contact based on Ti $(10 \mathrm{~nm}) / \mathrm{Al}(70 \mathrm{~nm}) / \mathrm{Ti}(10 \mathrm{~nm}) /$ $\mathrm{Au}(100 \mathrm{~nm})$ metal stack is firstly deposited by e-beam evaporation on the whole wafer backside, which is followed by rapid thermal annealing in $\mathrm{N}_{2}$ at $750{ }^{\circ} \mathrm{C}$ for $60 \mathrm{~s}$. Circular $\mathrm{Ni}(5 \mathrm{~nm}) / \mathrm{Au}(5 \mathrm{~nm})$ semitransparent Schottky contacts of $200 \mu \mathrm{m}$ in diameter are then deposited on the GaN active layer and patterned by lift-off technique. Next, all devices are passivated with a $150 \mathrm{~nm} \mathrm{SiO}_{2}$ layer deposited by plasma enhanced chemical vapor deposition. Finally, thick front 


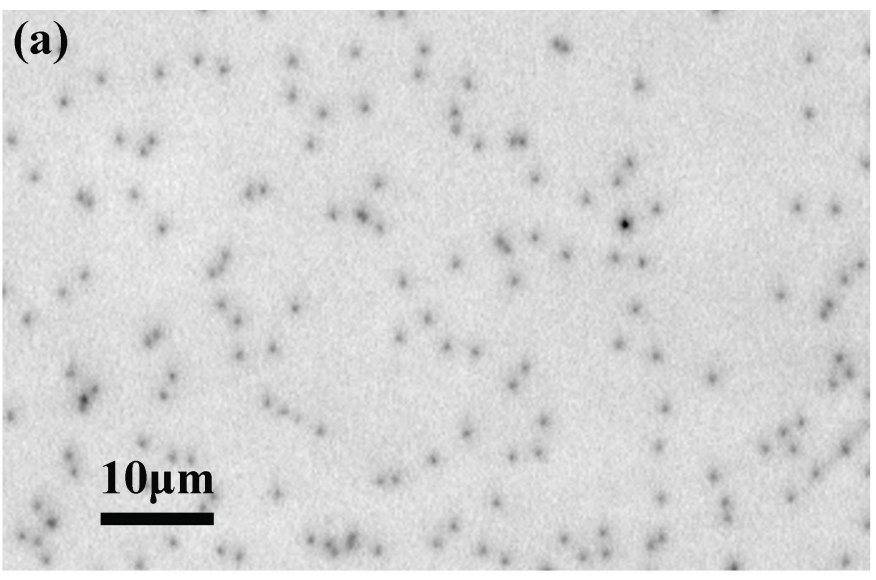

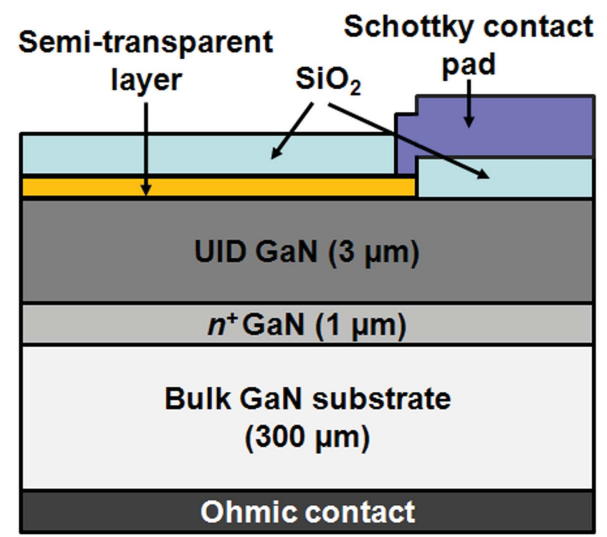

(b) (c)

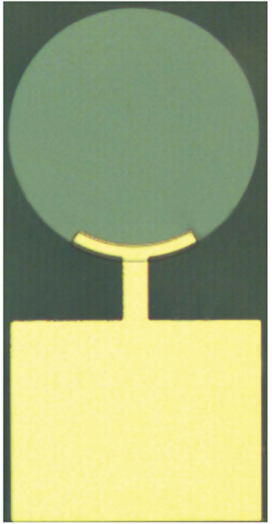

Fig. 1. (a) Panchromatic CL mapping image of the homoepitaxial GaN layer grown on bulk GaN substrate. (b) Schematic of the device structure. (c) Top view of one finished device.

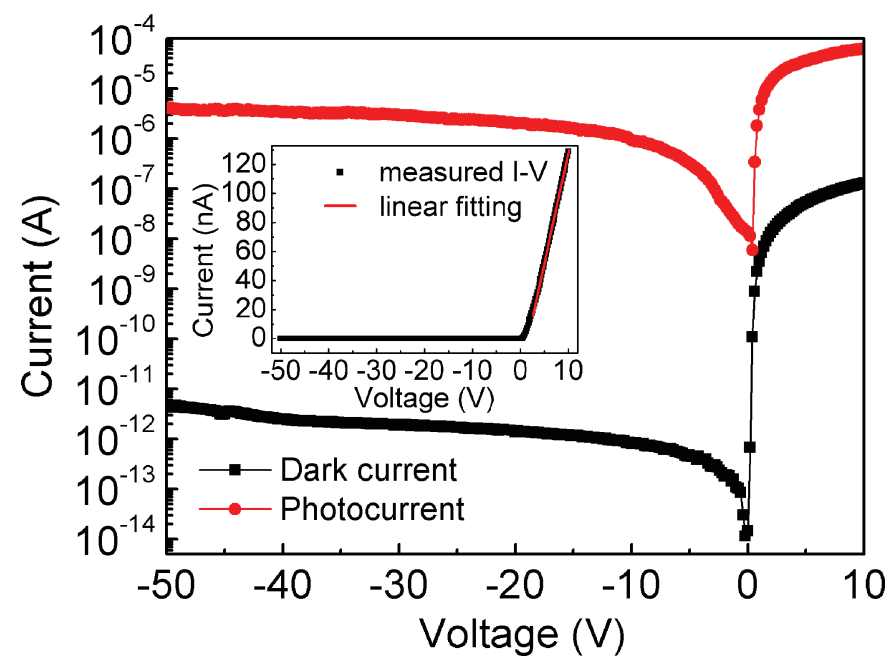

Fig. 2. $I-V$ characteristics of the PD measured under dark and under 365-nm light illumination. The optical power density of the 365-nm UV illumination is $\sim 5.5 \mu \mathrm{W} / \mathrm{mm}^{2}$. Inset: linear $I-V$ characteristic of the PD under dark.

contact pads based on $200 \mathrm{~nm} \mathrm{Ti} / \mathrm{Au}$ bi-layer is fabricated for easy electrical probing. Fig. 1(b) and (c) shows a schematic and a top view image of one finished device.

\section{RESUlts AND Discussion}

Fig. 2 shows the dark and photo-current curves of the PD measured at room temperature. Under reverse bias, the PD
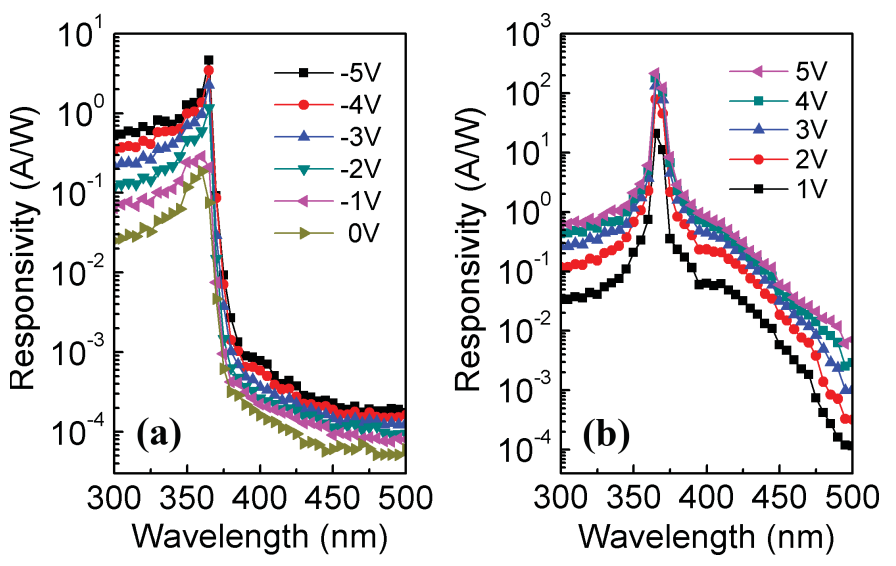

Fig. 3. Bias-dependent spectral response of the PD (a) under reverse, zero and (b) forward bias, respectively.

shows very low dark current. For example, the reverse dark current is $\sim 0.5 \mathrm{pA}$ at $-5 \mathrm{~V}$ bias, which corresponds to a dark current density of $1.4 \times 10^{-9} \mathrm{~A} / \mathrm{cm}^{2}$. Even at a high reverse bias of $-50 \mathrm{~V}$, the dark current is still smaller than $5 \mathrm{pA}$. The ultra-low reverse dark current achieved should be mainly attributed to the high crystalline quality of the GaN active layer. Meanwhile, it is interesting to find that the forward dark current of the PD is also quite low, which is $\sim 50 \mathrm{nA}$ at $5 \mathrm{~V}$ bias. As a result, a high photo-to-dark current ratio is observed in forward bias range, suggesting that the PD could also work like a photoconductor depending on the application needs. It is well known that high series resistance should be the main cause for the low forward current of a Schottky-type diode. Using a linear fit of the forward dark $I-V$ curve of the PD (inset of Fig. 2), the room-temperature resistivity of the $\mathrm{GaN}$ homo-epilayer is estimated as high as $6.0 \times 10^{7} \Omega \cdot \mathrm{cm}$. Based on our past study, the high resistivity obtained is likely caused by a change of local surface kinetics when the dislocation density of the growing $\mathrm{GaN}$ film is very low, so that much less impurities are incorporated into the GaN homo-epilayer [6], [8].

The spectral response characteristics of the Schottky barrier PD under reverse and zero bias are shown in Fig. 3(a), in which the PD works in conventional depletion mode. A sharp cutoff in the photo-response curves occurs at approximately $365 \mathrm{~nm}$, which corresponds to the bandgap energy of GaN. The UV/visible rejection ratio of the PD could be up to 6000 at $-5 \mathrm{~V}$ bias. Here, the UV/visible rejection ratio is defined as the photo-responsivity measured at $365 \mathrm{~nm}$ divided by that measured at $400 \mathrm{~nm}$. The thermally noise limited detectivity $\left(D^{*}\right)$ of the PD in depletion mode can be calculated by the following equation (9):

$$
D^{*}=R_{\lambda}\left(R_{0} A / 4 K T\right)^{1 / 2}
$$

where $R_{\lambda}$ is the zero-bias peak responsivity at $365 \mathrm{~nm}$, $R_{0} \sim 5.6 \times 10^{12} \Omega$ is dark impedance of the PD around zero bias, and $A$ is the device area. A detectivity as high as $6.3 \times 10^{13} \mathrm{~cm} \cdot \mathrm{Hz}^{1 / 2} / \mathrm{W}$ is then determined for the PD, which largely benefits from its relatively high responsivity and low dark current. Meanwhile, it is also found that the peak responsivity of the PD gradually increases as a function of reverse bias and reaches $4.6 \mathrm{~A} / \mathrm{W}$ at $-5 \mathrm{~V}$. The corresponding quantum 
efficiency well exceeds $100 \%$, indicating that an internal gain mechanism exists within the device. Our past study has shown that such internal gain is caused by photogenerated holes trapped at the semiconductor/metal interface as well as fieldinduced image-force lowering effect [10], [11].

When forward bias is applied to the PD, the operation status of the device switches to photoconductive mode. As shown in Fig. 3(b), the photo-response curve peaks at $\sim 365 \mathrm{~nm}$ with a maximum responsivity as high as $214 \mathrm{~A} / \mathrm{W}$ and a $\mathrm{UV} /$ visible rejection ratio more than 250 at $5 \mathrm{~V}$ bias. Interestingly, the photo-response of the PD drops sharply below $365 \mathrm{~nm}$, forming a narrow response peak with a FWHM of $\sim 6 \mathrm{~nm}$. This attractive observation means that the PD can be used for wavelength selective detection with high responsivity under forward bias. The formation mechanism of the narrow detection band is analyzed based on photoconductive gain. It has been reported that photoconductive gain in GaN-based photoconductor commonly originates from the increased conductive volume, which is modulated by photo-excitation [12]. Since $365 \mathrm{~nm}$ is the starting point for inter-band absorption, the optical absorption coefficient of GaN exactly around $365 \mathrm{~nm}$ is still not very high, so that the incident UV light could have a large penetration depth. The photo-carriers generated along the penetration path would form a conductive channel between the two contact electrodes, resulting a high photoconductive gain. When the wavelength of incident UV light is shorter than $365 \mathrm{~nm}$, the optical absorption coefficient of $\mathrm{GaN}$ would increase dramatically and quickly exceeds $10^{5} / \mathrm{cm}$. In this situation, most UV light would be absorbed by the upper GaN layer next to the Schottky electrode and the lower $\mathrm{GaN}$ layer would remain highly resistive. Since there is no sufficient conductivity modulation along the whole carrier traveling path, the photoconductive gain of the PD would drop quickly at shorter wavelength, leading to the narrow detection band centered at $\sim 365 \mathrm{~nm}$. Here it should be noted that for UVA-wavelength detection applications (especially for i-line at $365 \mathrm{~nm}$, which is most extensively used in UV curing industry) the narrow detection band of the PD means that less band-pass filter is required.

Finally, the temporal response of the PD is measured under the irradiation of a $365 \mathrm{~nm}$ UV light-emitting diode driven by a pulse generator. As shown in Fig. 4, the fall time of the PD (defined as the time for photocurrent to fall to $36.8 \%$ of its steady-state value) are $46 \mu \mathrm{s}$ and $60 \mu \mathrm{s}$ for $-5 \mathrm{~V}$ and $5 \mathrm{~V}$ bias respectively, which are close to the RC time constant of the PD, suggesting that the response speed of the PD is $\mathrm{RC}$ limited. Here it is worth noting that in spite of having a high series resistance $R \sim 5.6 \times 10^{7} \Omega$, the PD has fairly low capacitance $C \sim 0.8 \mathrm{pF}$ owing to its thick undoped $\mathrm{GaN}$ active layer. Meanwhile, the response time of the PD under forward bias is a little bit longer than that obtained under reverse bias, which should be caused by the additional photoconductive gain when the PD works in photoconductive mode.

\section{CONCLUSION}

A dual-operation-mode Schottky-type UV-PD is demonstrated on high-resistivity GaN homoepitaxial layer with low

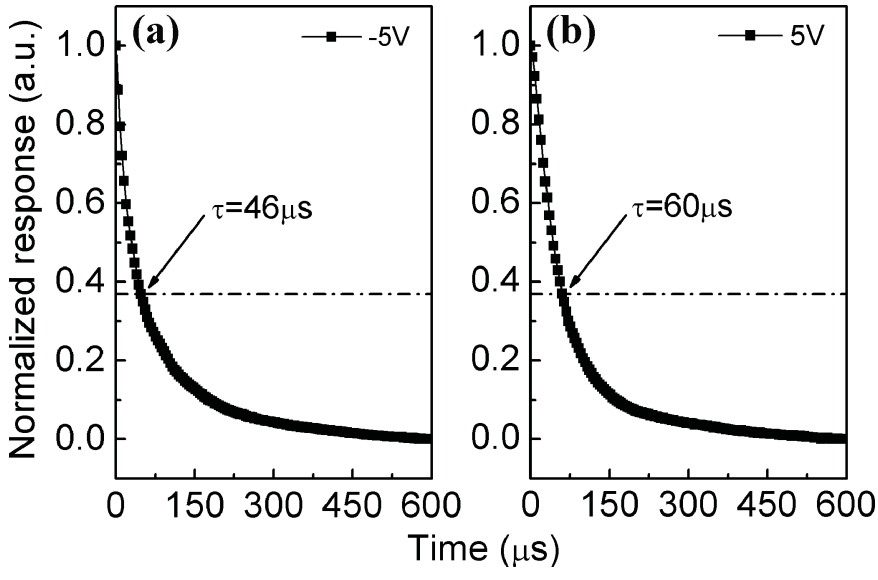

Fig. 4. Temporal response characteristics of the PD biased at (a) $-5 \mathrm{~V}$ and (b) $5 \mathrm{~V}$.

defect density. The operation mode of the PD is controlled by bias polarity. Under reverse and zero bias, the PD works in depletion mode with low dark current and high UV/visible rejection ratio. Under forward bias, the PD switches to photoconductive mode with high photo-responsivity and an attractive narrow detection band around $365 \mathrm{~nm}$. The PD exhibits reasonable response speed in both operation modes, which is likely RC limited.

\section{REFERENCES}

[1] M. Razeghi, "Short-wavelength solar-blind detectors-status, prospects, and markets," Proc. IEEE, vol. 90, no. 6, pp. 1006-1014, Jun. 2002.

[2] M. A. Khan, J. Kuznia, D. Olson, J. Van Hove, M. Blasingame, and L. Reitz, "High-responsivity photoconductive ultraviolet sensors based on insulating single-crystal GaN epilayers," Appl. Phys. Lett., vol. 60, no. 23, pp. 2917-2919, Jun. 1992.

[3] S. J. Chang, et al., "GaN-based MSM photodetectors prepared on patterned sapphire substrates," IEEE Photon. Technol. Lett., vol. 20, no. 22, pp. 1866-1868, Nov. 15, 2008

[4] N. Biyikli, T. Kartaloglu, O. Aytur, I. Kimukin, and E. Ozbay, "Highspeed visible-blind GaN-based indium-tin-oxide Schottky photodiodes," Appl. Phys. Lett., vol. 79, no. 17, pp. 2838-2840, Oct. 2001.

[5] Y. Zhang, et al., "Low-noise GaN ultraviolet p-i-n photodiodes on GaN substrates," Appl. Phys. Lett., vol. 94, no. 22, pp. 221109-1-221109-3, Jun. 2009.

[6] H. Shi, H. Lu, D. Chen, R. Zhang, and Y. Zheng, "High-resistivity GaN homoepitaxial layer studied by Schottky diode structure," Electron. Lett., vol. 45, no. 17, pp. 910-911, Aug. 2009.

[7] H. Lu, X. A. Cao, S. F. LeBoeuf, H. C. Hong, E. B. Kaminsky, and S. D. Arthur, "Cathodoluminescence mapping and selective etching of defects in bulk GaN," J. Cryst. Growth, vol. 291, no. 1, pp. 82-85, Apr. 2006.

[8] X. A. Cao, H. Lu, S. F. LeBoeuf, C. Cowen, S. D. Arthur, and W. Wang, "Growth and characterization of GaN PiN rectifiers on free-standing GaN," Appl. Phys. Lett., vol. 87, no. 5, pp. 053503-1-053503-3, Jul. 2005.

[9] T. Li, et al., "Low-noise back-illuminated $\mathrm{Al}_{x} \mathrm{Ga}_{1-x} \mathrm{~N}$-based p-i-n solar-blind ultraviolet photodetectors," IEEE J. Quantum Electron., vol. 37, no. 4, pp. 538-545, Apr. 2001.

[10] F. Xie, et al., "Low dark current and internal gain mechanism of GaN MSM photodetectors fabricated on bulk GaN substrate," Solid State Electron., vol. 57, no. 1, pp. 39-42, Mar. 2011.

[11] J. Burm and L. Eastman, "Low-frequency gain in MSM photodiodes due to charge accumulation and image force lowering," IEEE Photon. Technol. Lett., vol. 8, no. 1, pp. 113-115, Jan. 1996.

[12] E. Monroy, et al., "Si-doped $\mathrm{Al}_{x} \mathrm{Ga}_{1-x} \mathrm{~N}$ photoconductive detectors," Semicond. Sci. Technol., vol. 14, no. 8, pp. 685-689, Aug. 1999. 